Anuario Latinoamericano Ciencias Políticas

y Relaciones Internacionales vol. 5, 2017

pp. $237-251$

\section{Hacia nuevos retos. Aproximación a la participación electoral indígena en Paraguay}

DOI: $10.17951 /$ al.2017.5.237

\section{Towards new challenges. Approach to indigenous electoral participation in Paraguay}

\author{
Sara Mabel Villalba Portillo* \\ UNIVERSIDAD CATÓLICA, ASUNCIÓN, PARAGUAY \\ $\triangle$ saramabelvillalba@hotmail.com
}

\section{RESUMEN}

En este artículo se analizará la situación de los pueblos indígenas en Paraguay respecto al goce de su derecho a la participación politica, especificamente desde la esfera electoral. Para ello, se identificarán aspectos no formales y también normativos -desde el marco legislativo- que dificultan su intervención en procesos electorales. Asimismo, se contemplará el caso del primer movimiento político indígena que participará de manera autónoma en las elecciones generales de abril de 2018. A fin de contextualizar el tema a nivel regional, también se presentará un breve panorama sobre los partidos indígenas en América Latina.

PALABRAS CLAVE: participación electoral indígena, movimiento político indígena, partido indígena, Paraguay.

\section{ABSTRACT}

This article analyzes the situation of indigenous peoples in Paraguay regarding the enjoyment of their right to political participation, specifically in the electoral field. For this purpose, non-formal aspects as well as normative ones coming from the legislative framework will be identified, taking into account those which make it difficult to participate in electoral processes. In addition, the first indigenous political

\footnotetext{
* Doctorado en Procesos Políticos Contemporáneos y Máster Universitario en Ciencia Política (Universidad de Salamanca, España). Investigadora activa del Consejo Nacional de Ciencia y Tecnología (CONACYT) de Paraguay. Profesora titular de la Universidad Católica (UCA) y profesora contratada de la Universidad Nacional de Asunción (UNA). Máster en Ciencia Política y Licenciatura en Ciencias de la Comunicación (Universidad Nacional de Asunción, Paraguay). Principales líneas de investigación: mecanismos de democracia directa, acción colectiva, movilizaciones y participación indígena.
} 
movement to participate autonomously in the 2018 general elections will also be considered. In order to contextualize the issue at the regional level, a brief overview of the emergence of indigenous parties in Latin America and institutional factors that have driven the phenomenon will be presented.

KEYWORDS: indigenous electoral participation, indigenous political movement, Paraguay, indigenous party.

\section{Introducción}

La participación de los pueblos indígenas en Paraguay es un derecho consagrado en la misma Constitución Nacional. Sin embargo, no existen normativas ni medidas institucionales específicas que fomenten e impulsen la inserción real de los pueblos indígenas en el ámbito político. Actualmente ningún indígena ocupa un cargo electivo a nivel nacional y son muy escasas las autoridades electas en el nivel regional, incluso en los departamentos con mayor población indígena. En contraposición, en otros países latinoamericanos se ha registrado la participación creciente de indígenas en el electorado y la irrupción de partidos indígenas, que han logrado cargos electivos locales, regionales y nacionales.

En el presente artículo se analizará la situación de los pueblos indígenas en Paraguay respecto al goce de su derecho a la participación política, especialmente desde la esfera electoral. Se considerará la doble perspectiva del derecho al voto indígena y a ser electos para cargos a nivel departamental o nacional. En una primera parte se presentará y se delimitará el tema de la participación política indígena y se enfatizará en su aspecto electoral. A fin de contextualizar el tema a nivel regional, se presentará un breve panorama sobre la participación electoral y la emergencia de partidos indígenas en países de América Latina, así como los factores favorables para su desarrollo, desde la perspectiva institucionalista. En una segunda parte se abordará específicamente la participación electoral indígena en Paraguay y se analizará el marco legislativo pertinente. Asimismo, se contemplará la formación de movimientos políticos indígenas, así como del primer movimiento político indígena en el país que participará de forma autónoma en las elecciones generales, a ser realizadas en abril de 2018. Finalmente, en la tercera parte serán identificadas las principales limitaciones a la participación electoral indígena en Paraguay, teniendo en cuenta factores no formales y barreras legales e institucionales.

\section{Panorama general de la participación electoral indígena en América Latina}

Uno de los múltiples conceptos de la participación política señala que es el "conjunto de actividades voluntarias mediante las cuales los miembros de una 
sociedad participan en la selección de sus gobernantes y, directa o indirectamente, en la elaboración, ejecución, seguimiento y evaluación de la política gubernamental" (Sartori 1989). La última parte de la definición se refiere a las políticas públicas y la primera a la intervención de los ciudadanos en los procesos electorales. Entonces, se puede hacer referencia a la participación electoral como el involucramiento de los ciudadanos en las diferentes elecciones.

Para Nolhen (2004), la participación electoral es la más democrática e igualitaria porque incluye a la mayor cantidad de ciudadanos y se constituye en el canal principal de vinculación de los electores y sus representantes. Sostiene que "más allá del resultado electoral, más allá de la calidad moral, intelectual y política de los candidatos (...) la participación electoral es un hecho positivo en sí mismo, cuyo efecto inmediato beneficia al sistema político" (Nolhen 2004: 141).

La participación electoral de los pueblos indígenas -así como otras formas de participación política- se encuentra inserta en el marco de derechos políticos y su principal referente es el Estado. Posee dos aspectos complementarios: el sufragio activo referente al ejercicio del voto por parte de los indígenas; y el sufragio pasivo, o sea la existencia de candidaturas indígenas.

En América Latina las experiencias de participación electoral indígena son heterogéneas y diferenciadas debido a varios factores. Uno de éstos constituye la cantidad de población indígena respecto a la población total de un país. En países con alto porcentaje de población indígena es posible encontrar una presencia indígena más amplia y diversificada en cargos políticos y administrativos, mientras que en el caso de los países con menor población indígena, sus posibilidades de acceso a cargos electivos han sido considerablemente más reducidas. Sin embargo, el factor demográfico no es la única variable para impulsar la participación electoral de los indígenas, sino que existen otros aspectos de tipo institucional que han favorecido la constitución de partidos indígenas y su éxito en comicios electorales.

De acuerdo con el enfoque institucionalista, el Estado y la sociedad se afectan mutuamente y la democracia no solo depende de las condiciones económicas y sociales, sino también del diseño de las instituciones políticas (March y Olsen 1993: 13). Las instituciones determinan las oportunidades existentes en una sociedad y las organizaciones son creadas para aprovecharlas (North 1993: 18). Por tanto, la estructura institucional desempeña un papel clave para las innovaciones en una sociedad, marco donde se sitúan los partidos indígenas. Uno de los factores que propició la creación de estos partidos en América Latina fue la codificación de derechos constitucionales especiales para pueblos indígenas y los cambios en la legislación electoral que les facilitaron el ingreso a los sistemas de partidos nacionales y en ciertos casos determinaron su éxito electoral.

Algunas investigaciones de corte empírico señalan que existen circunstancias particulares en la estructura política que permiten la emergencia y la viabilidad electoral de los partidos indígenas en América Latina (Yashar 1998;
Hacia nuevos retos. Aproximación a la participación electoral indígena en Paraguay

Sara Mabel Villalba Portillo 
Van Cott 2003; Van Cott 2005; Marmon y Kramer 2006; Alcántara y Marenghi 2007; Krzywicka 2011). Entre estos factores institucionales se incluyen los sistemas electorales inclusivos, la descentralización, el reconocimiento legal de los derechos indígenas, la permeabilidad del sistema de partidos políticos. De acuerdo con los estudios mencionados, estos factores posibilitaron el éxito electoral principalmente de partidos indígenas de Bolivia, Ecuador, Venezuela y Colombia.

\section{Participación electoral indígena en Paraguay}

La población indígena en Paraguay asciende actualmente a 112.848 personas, según el último Censo Nacional de Población y Viviendas para Pueblos Indígenas del 2012. Son 19 pueblos indígenas, agrupados en cinco familias lingüísticas y cada pueblo posee una lengua propia. Asimismo, junto con el español, el guaraní es idioma oficial de Paraguay y utilizado por aproximadamente el 90\% de la población.

En cuanto a su distribución demográfica, en la Región Occidental del país se registra un elevado porcentaje de población indígena en relación con el resto de la población. Específicamente en los departamentos de Boquerón, Alto Paraguay y Presidente Hayes, la población indígena alcanza el 40\%, el 37\% y el $24 \%$ respectivamente, de acuerdo con datos del III Censo Nacional de Población y Viviendas para Pueblos Indígenas. En la Región Oriental existen minorías significativas de población indígena en los departamentos de Amambay (12,1\%), Canindeyú $(11,1 \%)$ y Caaguazú $(8 \%)$.

Pese a la importancia cultural e histórica de los pueblos indígenas en la formación de la sociedad paraguaya, los principales partidos políticos del $\mathrm{Pa}$ raguay: el Partido Liberal Radical Auténtico (PLRA) y la Asociación Nacional Republicana (ANR) no hacen referencia alguna a los indígenas ni en su declaración de principios ni en sus idearios. Sin embargo, en las elecciones celebradas en los años posteriores al régimen dictatorial de Alfredo Stroessner (19541989) hasta nuestros días, algunos partidos políticos han incluido a indígenas en su lista de candidatos, principalmente para la ocupación de cargos en las Juntas Municipales y Departamentales. Como ejemplo pueden mencionarse casos puntuales como el de René Ramírez, indígena Enlhet, del departamento de Presidente Hayes, electo convencional constituyente en representación del PLRA (Harder Host 2001: 135). Asimismo, Susana Pintos y Severo Flores, del pueblo Guaraní Occidental, Cirilo Pintos, del pueblo Nivaclé, y Juan Giménez, del pueblo Enxet, fueron concejales municipales de Mariscal Estigarribia por el Partido Encuetro Nacional (PEN), en el periodo 2001-2006. También Hipólito Acevei, del pueblo Guaraní Occidental, fue concejal departamental en Boquerón, por el PEN, en el periodo 1998-2003. Además, un indígena fue concejal municipal por el PLRA en el distrito de Minga Porá en el periodo 2003-2008. 
En las últimas elecciones nacionales de 2013, el movimiento Kuña Pyrenda tuvo tres candidatas indígenas al Senado, dos candidatas al PARLASUR y cinco candidatas a la Junta Departamental de Boquerón. La Alianza Frente Guazú postuló a un candidato a gobernador y nueve candidatos a miembros de Junta Departamental de Boquerón, y el Movimiento Independiente Constitucionalista en Alianza (MICA) tuvo un candidato a senador, según el informe final de la Misión de Observación Electoral de la Unión Europea en Paraguay (MOEUE 2013). El documento señala, además, que "ningún partido o movimiento político se atribuyó la representación de los intereses indígenas ni hubo visibilidad de los candidatos indígenas durante la campaña” (MOEUE 2013: 29).

En efecto, la relación de los indígenas con los partidos políticos continúa siendo relativamente marginal, más allá de ciertas situaciones coyunturales y de las relaciones circunstanciales entre políticos e indígenas. A priori, una de las características de la resistencia indígena a la imposición de pautas y valores de parte de la sociedad paraguaya es su no-participación política en ella. "Esta postura de los pueblos indígenas normalmente se ha manifestado en una indiferencia hacia los partidos políticos y a sus esfuerzos de proselitismo dentro de las comunidades indígenas" (Robins 1999: 136).

De hecho, el contacto directo de los indígenas con los partidos políticos era escaso durante la dictadura stronista, puesto que en general los políticos no tenían necesidad de hacer proselitismo entre los indígenas, dado que la victoria del partido oficialista, la ANR, estaba asegurada. Sin embargo, esta situación cambió radicalmente luego de la dictadura, cuando los pueblos indígenas iniciaron varias formas de participación política, cuyo principal objetivo eran demandas de tierra y de mejores condiciones de vida. Por su parte, los políticos se dieron cuenta del caudal de votos que representaban las comunidades indígenas y comenzaron a hacer campañas y afiliarlos. Las agrupaciones políticas que han marcado presencia en las comunidades fueron los dos partidos tradicionales: ANR y PLRA, además del entonces novel Partido Encuentro Nacional. Las estrategias principales utilizadas para "ganar" el voto indígena fueron la provisión de víveres y alcohol, la influencia en líderes y maestros de las comunidades, además de las promesas de una serie de mejoras viales o de infraestructura en las comunidades, que, en su mayoría, no fueron cumplidas (Kidd 1997: 32-43).

\section{Marco legal para la participación electoral indígena}

En los últimos decenios se ha fortalecido el marco legal en favor de los derechos de los pueblos indígenas, especialmente en el nivel internacional y con repercusiones favorables en el ámbito nacional. Entre estos derechos se contempla el derecho a la participación.

El Convenio 169 sobre pueblos indígenas y tribales en países independientes se constituye en el instrumento jurídico más importante de salvaguarda de los derechos indígenas. Fue adoptado por la Organización Internacional
Hacia nuevos retos. Aproximación a la participación electoral indígena en Paraguay

Sara Mabel Villalba Portillo 
del Trabajo (OIT), en 1989. Si bien no se refiere específicamente a la "participación política indígena", señala que los pueblos indígenas deben gozar "sin discriminación de los derechos generales de ciudadanía” (Art. 4) y que los miembros de dichos pueblos deben "ejercer los derechos reconocidos a todos los ciudadanos del país" (Art. 8). A nivel nacional, en Paraguay, se encuentra vigente la Ley 294/94 que ratifica el Convenio 169.

Asimismo, la Convención Internacional sobre la Eliminación de todas las Formas de Discriminación Racial, aprobada por la ONU en 1969, estipula en su artículo 5 que los Estados partes se comprometen a prohibir y eliminar la discriminación racial en todas sus formas y a garantizar el derecho de toda persona a la igualdad ante la ley, sin distinción de raza, color y origen nacional o étnico, particularmente en el goce de los derechos políticos, "en particular el de tomar parte en elecciones, elegir y ser elegido, por medio del sufragio universal e igual, el de participar en el gobierno y en la dirección de los asuntos públicos en cualquier nivel, y el de acceso, en condiciones de igualdad, a las funciones públicas". Paraguay es signatario de esta convención, lo cual se traduce en la Ley $\mathrm{N}^{\circ} 2128 / 03$.

Aunque no es vinculante, la Declaración de los Derechos de los Pueblos Indígenas también establece en su artículo 5 que "Los pueblos indígenas tienen derecho a conservar y reforzar sus propias instituciones políticas, jurídicas, económicas, sociales y culturales, manteniendo a la vez su derecho a participar plenamente, si lo desean, en la vida política, económica, social y cultural del Estado". Asimismo, el artículo 18 sostiene que "Los pueblos indígenas tienen derecho a participar en la adopción de decisiones en las cuestiones que afecten a sus derechos, por conducto de representantes elegidos por ellos de conformidad con sus propios procedimientos, así como a mantener y desarrollar sus propias instituciones de adopción de decisiones".

Paraguay reconoce el derecho constitucional a la participación de los pueblos indígenas y sostiene en el artículo 65 que "Se garantiza a los pueblos indígenas el derecho a participar en la vida económica, social, política y cultural del país, de acuerdo con sus usos consuetudinarios, ésta Constitución y las leyes nacionales”. Además, la ley 904/81 Estatuto de las Comunidades Indígenas, declara que "Esta Ley tiene por objeto la preservación social y cultural de las comunidades indígenas, la defensa de su patrimonio y sus tradiciones, el mejoramiento de sus condiciones económicas, su efectiva participación en el proceso de desarrollo nacional...”.

Asimismo, respecto al derecho a la participación política, la Ley 5/92 aprueba la adhesión de Paraguay al Pacto Internacional de Derechos Civiles y Políticos, que en su artículo 5 estipula que “Todos los ciudadanos gozarán,(...), y sin restricciones indebidas, de los siguientes derechos y oportunidades: a) Participar en la dirección de los asuntos públicos, directamente o por medio de representantes libremente elegidos; b) Votar y ser elegidos en elecciones periódicas, auténticas, realizadas por sufragio universal e igual y por voto secreto que garantice la libre expresión de la voluntad de los elec- 
tores; c) Tener acceso, en condiciones generales de igualdad, a las funciones públicas de su país".

Dado que los derechos humanos son interdependientes y están relacionados entre sí, el derecho a la participación política se encuentra estrechamente vinculado a todos los demás derechos políticos. En la Tabla no 1 pueden apreciarse los principales instrumentos legales que salvaguardan el derecho de los pueblos indígenas a la participación en general y también sus derechos políticos en Paraguay.

En líneas generales, Paraguay ha ratificado los más relevantes tratados internacionales para garantizar elecciones periódicas democráticas, así como la no discriminación en el goce y ejercicio de los derechos políticos.

\begin{tabular}{|c|c|c|c|}
\hline Instrumento & Carácter & Ámbito & Año \\
\hline $\begin{array}{l}\text { Pacto Internacional de Derechos } \\
\text { Civiles y Políticos }\end{array}$ & Vinculante & Internacional & 1966 \\
\hline $\begin{array}{l}\text { Convención Internacional sobre la } \\
\text { Eliminación de todas las Formas de } \\
\text { Discriminación Racial }\end{array}$ & Vinculante & Internacional & 1965 \\
\hline $\begin{array}{l}\text { Convenio } 169 \text { sobre pueblos indígenas } \\
\text { y tribales en países independientes }\end{array}$ & Vinculante & Internacional & 1989 \\
\hline $\begin{array}{l}\text { Declaración de los Derechos de los } \\
\text { Pueblos Indígenas }\end{array}$ & No vinculante & Internacional & 2007 \\
\hline Constitución Nacional & Vinculante & Nacional & 1992 \\
\hline $\begin{array}{l}\text { Ley } 904 / 81 \text { Estatuto de las } \\
\text { Comunidades Indígenas }\end{array}$ & Vinculante & Nacional & 1981 \\
\hline $\begin{array}{l}\text { Ley N }{ }^{\circ} 05 / 92 \text { "Que aprueba la } \\
\text { adhesión de la República al Pacto } \\
\text { Internacional de Derechos Civiles y } \\
\text { Políticos, adoptados durante el XXI } \\
\text { periodo de sesiones de la Asamblea } \\
\text { General de la Organización de las } \\
\text { Naciones Unidas, en la ciudad de } \\
\text { Nueva York el } 16 \text { de diciembre 1966" }\end{array}$ & Vinculante & Nacional & 1992 \\
\hline $\begin{array}{l}\text { Ley No } 2128 / 92 \text { "Que aprueba la } \\
\text { Convención Internacional sobre la } \\
\text { Eliminación de todas las Formas de } \\
\text { Discriminación Racial" }\end{array}$ & Vinculante & Nacional & 1992 \\
\hline $\begin{array}{l}\text { Ley 234/93 "Que ratifica por parte del } \\
\text { gobierno paraguayo el Convenio } 169 \\
\text { de la OIT" }\end{array}$ & Vinculante & Nacional & 1993 \\
\hline
\end{tabular}

Fuente: Elaboración propia.
Hacia nuevos retos.

Aproximación a la participación electoral indígena en Paraguay

Sara Mabel Villalba Portillo
Tabla 1.

Marco legal del derecho a la participación de los pueblos indígenas en Paraguay 
Sin embargo, en el marco legal electoral no se menciona de manera específica a los indígenas. ${ }^{1} \mathrm{Ni}$ siquiera en la Constitución Nacional se abordó de forma diferenciada el derecho a la participación de los pueblos indígenas (Prieto 2013: 46).

\section{Surgimiento de movimientos políticos indígenas en Paraguay}

En 2000 y 2001 surgieron dos organizaciones políticas indígenas en Paraguay: el Movimiento 19 de Abril (M19) y el Movimiento 11 de Octubre (MIO). Ambos movimientos políticos ${ }^{2}$ se originaron en los departamentos de Presidente Hayes y Boquerón, respectivamente, zonas con marcada población indígena.

El Movimiento Indígena 19 de Abril fue la primera organización indígena de carácter político que se originó en el Paraguay. Se inició en octubre de 2000 en el departamento de Presidente Hayes, en el seno de la Coordinadora de Líderes Indígenas del Bajo Chaco (CLIBH). Adoptó el nombre "19 de abril" porque esa fecha tiene un "significado de protesta por los abusos cometidos por los gobiernos en contra de los pueblos indígenas". ${ }^{3}$ El M19 fue integrado por indígenas de los pueblos Lengua, Enxet, Nivaclé, Sanapaná, Toba Qom y Angaité. Se constituyó en una organización política indígena de carácter regional, con el objetivo de defender las reivindicaciones indígenas, relacionadas con el cese de la explotación en las estancias, la sustracción de tierras ancestrales y el avasallamiento de su cultura. Según declaraciones de los indígenas publicadas en la prensa, el M19 consistía en una alternativa política para los indígenas, ante la escasa apertura de los partidos políticos tradicionales, quienes solamente se interesan por ellos en época de elecciones. Su dirección estuvo a cargo de un Consejo Político Religioso Indígena, integrado por 27 líderes de comunidades chaqueñas.

1 El marco regulatorio de las elecciones en Paraguay incluye a: 1) la Constitución de 1992; 2) el Código Electoral (Ley $N^{\circ} 834 / 96$ ); 3 ) la Ley de Justicia Electoral (Ley $N^{\circ}$ 635/95); y 4) las resoluciones del Tribunal Superior de Justicia Electoral. Además, el Código Penal (Ley N 1160/07) sanciona la violación del secreto de voto, la compra de votos, los casos de intimidación, voto fraudulento, falsificación de resultados y violaciones del Código Electoral.

2 Para los fines de este artículo, se entiende movimiento político como la agrupación de personas que a través de los mecanismos electorales pretende acceder al poder. Según la legislación paraguaya, se constituye en una agrupación que aún no ha cumplido todos los requisitos pertinentes exigidos por el Tribunal Electoral de la Capital para conformarse como partido político. Es considerada una entidad de carácter transitorio. No obstante, de acuerdo con el artículo 11 de la Ley Electoral, el movimiento político es reconocido como persona jurídica de derecho público interno desde el momento que es reconocido por la Justicia Electoral. Según el Art. 88 del Código Electoral, "se aplicarán a los movimientos políticos, en lo que fuere pertinente, todas las disposiciones relativas a los partidos políticos".

3 El 19 de abril de 1940 grupos indígenas habían protestado por no ser invitados a participar del Primer Congreso Indigenista Interamericano, realizado en Pátzcuaro, México. 
El otro movimiento indígena que surgió en la misma época fue el Movimiento 11 de Octubre (MIO), presentado oficialmente en Filadelfia, departamento de Boquerón, en abril de 2001. El nombre fue elegido porque se constituye en el día previo al descubrimiento de América. La organización surgió a instancias de líderes indígenas con trayectoria política en los partidos tradicionales, especialmente en el Partido Encuentro Nacional (PEN). De hecho, tres miembros activos del MIO ocuparon cargos en la Junta Municipal, en representación del PEN, en el municipio de Filadelfia y uno de ellos integró la Junta Departamental de Boquerón (Villalba 2007). Algunos objetivos del MIO fueron "fortalecer el protagonismo diferenciado de los pueblos indígenas en la vida cotidiana e institucional dentro del departamento de Boquerón; encaminar educación cívica en las comunidades y diseñar colectivamente estructura y formas de participación política de los pueblos indígenas del departamento. Participar con candidatos propios en las elecciones municipales, departamentales y nacionales y diseñar la gestión participativa de los pueblos indígenas en los órganos de gobierno local, departamental y nacional". El MIO fue integrado por indígenas de los pueblos Nivaclé, Guaraní, Angaite, Ayoreo, Guaraní Nandeva, Lengua y Maskoy. Su estructura organizativa incluyó a un coordinador general, un asesor, un secretario y cuatro miembros del Comité Político, además de un grupo de acompañamiento.

Tanto el MIO como el M19 participaron en las elecciones nacionales en 2003 para pugnar por cargos en los departamentos de Boquerón y Presidente Hayes. Posteriormente, en los comicios de 2008, participó únicamente el M19. Sin embargo, en ambas ocasiones, ninguna de las dos agrupaciones pudo presentarse de manera autónoma, sino bajo auspicios de otros partidos políticos. El motivo fue que, si bien poseían personería jurídica, aún no contaban con el reconocimiento de la Justicia Electoral porque no pudieron reunir todos los requisitos exigidos por la Ley Electoral (estatutos, inscripción en el Registro Cívico Permanente, entre otros requerimientos).

En 2003, el M19 se presentó bajo auspicios del Partido Patria Libre y en el marco de una coalición denominada Izquierda Unida, que aglutinó a organizaciones sociales y políticas como: Partido de los Trabajadores, Corriente Gremial Campesina, Movimiento por la Igualdad, Movimiento de Recuperación Democrática, Corriente Sindical y Social y Corriente Vecinal Popular. Por su parte, el MIO se presentó bajo auspicios del Partido País Solidario pugnando por los cargos de gobernador, diputado y concejales departamentales ${ }^{4}$. En las elecciones de 2008, el M19 participó bajo la chapa del Partido de los Trabajadores. En todos los casos mencionados, los resultados fueron adversos y obtuvieron escasos votos. La causa de esta situación fue atribuida por los mismos indígenas y observadores externos (Villalba 2007) principalmente a las extendidas prácticas clientelistas y prebendarías de los partidos políticos

4 Los candidatos indígenas habían sido elegidos por votación durante una asamblea, donde participaron 200 líderes de comunidades.
Hacia nuevos retos.

Aproximación a la participación electoral indígena en Paraguay

Sara Mabel Villalba Portillo 
tradicionales, entre otros factores como la escasez de recursos y la ausencia de una estructura organizativa de movimiento social precedente a la formación de una organización política con fines electorales.

\section{Primer movimiento indígena autónomo en Paraguay}

En el 2017 y con miras a las elecciones nacionales de abril de 2018 se conformó el Movimiento Político Indígena Plurinacional del Paraguay (MPIP). Este se constituye en el primer movimiento político indígena que competirá de manera autónoma -esto es sin el auspicio de otro partido- y por cargos de nivel nacional como la Cámara de Senadores. La lista está encabezada por el arquitecto indígena Gerónimo Ayala, del pueblo Mbya Guaraní, que aspira a convertirse en el primer senador indígena en la historia del país.

Para el reconocimiento oficial del movimiento por parte de la Justicia Electoral, en tres meses juntaron más de las 15.000 firmas requeridas por la legislación electoral. La colecta de firmas fue realizada por indígenas de diversos pueblos en varias ciudades del país. De manera previa, dos años antes, realizaron consulta con líderes de los pueblos indígenas en todo el país. Entre las reivindicaciones principales de la organización figuran: „la democracia intercultural" y la protección de los derechos indígenas de acceso a la tierra, educación y salud, así como la protección del medio ambiente y la generación de comunidades autosustentables.

\section{Limitaciones a la participación electoral indígena en Paraguay}

Las dificultades para el ejercicio de la participación política indígena constituyen una situación manifestada hace décadas en Paraguay. Especialmente en las últimas elecciones nacionales de 2013 se registraron numerosas falencias, estipuladas por los grupos de observadores internacionales en el informe del MOEUE 2013. En el citado documento se considera a los pueblos indígenas como actores ciudadanos con altos niveles de exclusión de la participación política en el país. Además, el texto señala que las comunidades indígenas adolecen de elevada precariedad en términos de educación cívica y se encuentran expuestos a la coacción electoral y a la mercantilización del voto.

En suma, existen numerosas barreras para la participación política efectiva de los pueblos indígenas en el país, las cuáles resultan más notorias en el ámbito electoral. Estos factores están relacionados con el "modo de hacer política" en Paraguay y afectan no solamente a los indígenas, sino a gran parte de la población electora.

Además de las limitaciones no formales para la participación política, también existen vacíos legales e incluso algunas disposiciones del marco electoral 
que desalientan u obstaculizar la participación electoral indígena. Al respecto, el informe del MOEUE 2013 da cuenta de que la legislación electoral no menciona a los pueblos indígenas y la presencia indígena en cargos electivos de nivel nacional sigue siendo nula. Entre los factores no formales que dificultan la participación electoral indígena pueden resumirse tres principales: mercantilización del voto indígena, escasa comprensión del procedimiento de votación y dificultades para llegar a los locales de votación. La mercantilización del voto indígena se manifiesta principalmente a través de prácticas prebendarias en la época de elecciones, específicamente a través de la compra de votos a cambio de ciertos beneficios o promesas. Los operadores políticos entregan víveres, medicamentos, bebidas alcohólicas, camisetas con leyendas partidarias y dinero a los líderes o a otros miembros de la comunidad, a cambio de sus votos. Las necesidades apremiantes que padecen estos pueblos constituyen el caldo de cultivo propicio para el afianzamiento de estas prácticas.

Esta había sido ya una de las principales denuncias de las organizaciones indigenistas durante los comicios nacionales celebrados en 1993. Estas denuncias han afectado fundamentalmente al Partido Colorado, pero los demás partidos también ejercen las mismas prácticas. Por ejemplo, para las internas del Partido Colorado en las mesas de votación no se exigían los documentos de afiliación, sino únicamente las cédulas de identidad, las cuales eran compradas a los indígenas. Las comunidades más afectadas por estas prácticas fueron de los pueblos Nivaclé, Guaraní, Guaraní Ñandeva, en los departamentos de Presidente Hayes y Boquerón (DIM 1993: 4-5).

Esta cuestión también fue constatada en las últimas elecciones nacionales de 2013. Al respecto, el informe del MOEUE 2013 indica que los observadores electorales fueron testigos directos de este tipo de prácticas. Además, menciona la realización de encierros de votantes indígenas durante los días previos a las elecciones. Los denominados "corralones" son lugares cercados al aire libre donde se da de comer y beber a indígenas hasta que llega el día y la hora de votar. Posteriormente, estas personas son devueltas a sus comunidades de origen después de emitir su voto, que ha sido previamente comprado. La práctica mencionada fue observada en siete ocasiones en los distritos de Mariscal Estigarribia y Loma Plata (departamento de Boquerón) y en el distrito Teniente Irala Fernández (departamento de Presidente Hayes). Si bien el encierro en "corralones" no se produce en contra de la voluntad de los indígenas, la MOEUE “considera esta práctica como atentatoria contra la dignidad humana, por la manera en que se reduce a los votantes a mercancía electoral, y la condena con firmeza" (MOEUE 2013: 6, 21).

Asimismo, la dificultad para la comprensión del proceso de votación por parte de los indígenas se constituye en otra barrera no formal para su participación electoral. Los principios y mecanismos de la democracia representativa constituyen una cuestión ajena a la cultura de los pueblos indígenas. Originalmente, las diferentes comunidades indígenas participan en un modelo de democracia comunitaria centrada en el consenso y no en el instrumento del
Hacia nuevos retos. Aproximación a la participación electoral indígena en Paraguay

Sara Mabel Villalba Portillo 
voto, por tanto lo colectivo prevalece sobre lo individual (Domínguez y Santiago 2014: 97-98). Además, esta situación implica que los pueblos indígenas en general y en Paraguay deben convivir con dos sistemas de organización política diferente, uno al interior de sus comunidades y otro en su relacionamiento con el resto de la sociedad paraguaya y en condiciones de desventaja (Prieto 2013: 41-42). Por ende, en lo que respecta a su participación en el ámbito electoral los pueblos indígenas deben realizar un esfuerzo mucho mayor para la sola compresión de la naturaleza del Estado y las reglas que rigen su funcionamiento. Además, se encuentran obligados a competir en un sistema electoral ideado en un contexto socio-histórico totalmente ajeno a sus culturas. En consecuencia para su adaptación y comprensión deben realizar un esfuerzo mayor al de otros ciudadanos paraguayos no indígenas. También existe un desconocimiento o escaso manejo del español que impide que puedan comprender a cabalidad las complejas leyes electorales (Prieto 2013: 46,60).

Otro problema sumado a las limitaciones mencionadas constituye que los locales en los que se ejerce la votación no tienen una localización especial considerando las características de la población indígena (López 2015: 19). Al respecto, el informe del MOEUE 2013 indica que la alta concentración de mesas receptoras de voto en locales de votación implica que muchos electores tengan que recorrer largas distancias para ejercer su derecho a voto. Habilitar más mesas receptoras en las cercanías de núcleos de población rural mitigaría el problema (MOEUE 2013: 8).

El análisis del surgimiento y la configuración de los movimientos indígenas en áreas electorales se plantea, con pocas excepciones, desde el supuesto de que esta emergencia ocurre a contra corriente de las disposiciones del régimen electoral. Paraguay es un ejemplo claro de esta situación. En esta línea, también existen factores de tipo legal e institucional que constituyen limitaciones a la participación electoral indígena en el país. Una primera dificultad es la ausencia de cualquier acto afirmativo o discriminación positiva a favor de los pueblos indígenas en la legislación electoral. Esto significa que, a pesar de todas las limitaciones propias de un grupo vulnerable, los movimientos políticos indígenas deben competir en igualdad de condiciones con los demás partidos y movimientos de la sociedad no indígena, incluyendo partidos tradicionales y mayoritarios. Además, en Paraguay no existen ni circunscripciones indígenas ni cuotas especiales como en otros países. Las circunscripciones electorales especiales para la población indígena constituyen una manera de garantizar la representación e incentivan la creación de partidos indígenas. Una vez instaladas, estas circunscripciones también permitieron expandir la representación por encima del nivel mínimo. Por ejemplo, en Colombia y Venezuela, después de obtener las curules asignadas en las circunscripciones especiales, compitieron exitosamente por curules no reservadas (Van Cott 2003; Martínez 2004).

Las cuestiones mencionadas denotan que el sistema electoral paraguayo no es inclusivo. En efecto, en un sistema electoral inclusivo se estipula el establecimiento de menores umbrales para la representación, lo cual implica 
requerimientos mínimos de votos para la asignación de curules. Las fórmulas de asignación de curules a los partidos que ganen votos deben correlacionarse con la formación y la viabilidad electoral de los nuevos partidos (en este caso los partidos indígenas). Se parte de la conjetura de que los partidos indígenas obtendrían pocos votos. Por lo tanto, los umbrales menores les permitirán tener acceso a cargos políticos y subsidios estatales partidarios, que servirían para aumentar su votación en las siguientes elecciones (Van Cott 2003: 32).

El Código Electoral paraguayo establece ingentes requisitos para el reconocimiento de los partidos políticos. Además del acta de fundación, la declaración de principios, los estatutos y la nómina de la directiva para su reconocimiento por la Justicia Electoral, los partidos deben contar con un número de afiliados no inferior al 0,5\% de los votos válidos emitidos en las últimas elecciones al Senado y deben probar que cuentan con organizaciones en Asunción y en, por lo menos, cuatro ciudades capitales departamentales del país, lo que dificulta el registro de partidos por parte de grupos regionalmente concentrados, minorías y poblaciones indígenas (MOEUE 2013: 18). Asimismo, el Código Electoral no permite la formación de partidos regionales, sino únicamente movimientos políticos, de carácter transitorio, para la presentación de candidaturas a elecciones departamentales y municipales. Esta situación implica la necesidad de más recursos, pues para cada elección debe realizarse todo el proceso de reconocimiento partiendo de cero. Otra limitación del Código Electoral es que no cuenta con ninguna cláusula especial sobre la inscripción y el voto de los indígenas. Esto significa que el Padrón Electoral no hace ninguna distinción respecto de la condición de los sufragantes y lo mismo ocurre con los resultados de las votaciones. Este desconocimiento total de información sobre el voto indígena y su invisibilización limita también la capacidad de negociación de los pueblos indígenas en el ámbito partidario y en el sistema político en general (Prieto 2013: 85-86).

\section{Conclusiones}

La configuración de las instituciones estatales ha constituido uno de los factores que ha condicionado la formación de los partidos indígenas, de acuerdo a la bibliografía especializada. Esta misma situación se ha registrado en Paraguay, donde las diversas barreras normativas impuestas por el sistema electoral dificultan el surgimiento, la consolidación y el éxito electoral de los movimientos políticos y partidos indígenas.

Si bien, uno de los motivos mencionados por los indígenas para la creación de movimientos propios consiste en el debilitamiento de los partidos políticos como instituciones de la democracia representativa, hasta ahora tampoco han podido encontrar otras salidas más factibles que la formación de partidos y movimientos propios que puedan representarlos
Hacia nuevos retos. Aproximación a la participación electoral indígena en Paraguay

Sara Mabel Villalba Portillo 
Un aspecto de la representatividad a ser considerado es el factor exógeno que consiste en la repercusión que podrían tener estas organizaciones políticas indígenas en el ámbito nacional en Paraguay. Para muchos indígenas la conformación de una organización y de un discurso político propios los vuelve "visibles" para la sociedad envolvente y principalmente visibilizaría sus demandas de tierra y de mejores condiciones de vida.

No obstante, existen aún múltiples y complejos desafíos en torno de la participación electoral indígena en Paraguay. A pesar de los avances en materia de derechos y también de participación política, todavía se requiere promover acciones que aseguren la integración de los pueblos indígenas en el ejercicio de la política nacional, tanto en sus roles como electores y candidatos.

El hecho de que la participación electoral indígena en Paraguay no cuente con espacio mediático o académico no necesariamente implica que dicho proceso no sea relevante para el sistema político o la sociedad de un determinado país, más aun teniendo en cuenta que la Constitución paraguaya reconoce los derechos de estos pueblos.

\section{Bibliografía}

Alcántara M., Marenghi P. (2007), Los partidos étnicos de América del Sur: algunos factores que explican su rendimiento electoral, en: Pueblos indígenas y política en América Latina, Martí i Puig, S. (ed.), Fundación CIDOB, Barcelona.

Freidenberg F. (2008), El sueño frustrado de la gobernabilidad: instituciones, actores y política informal en Ecuador, Fundación CIDOB, Barcelona.

Krzywicka K. (2011), Situación jurídica de los pueblos indígenas en Venezuela. Dilemas de representación y participación, "Revista del CESLA", no 14, pp. 73-107.

Kidd S. (1997), Tierra, política y chamanismo benévolo. Los indígenas Enxet en un Paraguay democrático, "Suplemento Antropológico", vol. XXXII, no 1-2, pp. 9-59.

López M. (2015), Acceso a los derechos electorales. Paraguay, European University Institute, Florence.

Mainwaring S, Scully T. (1996), La construcción de instituciones democráticas. Sistema de partidos en América Latina, Corporación de Investigaciones Económicas para Latinoamérica, Santiago.

March J., Olsen J. (1993), El nuevo institucionalismo: factores organizativos de la vida política, "Zona Abierta", no 63-64, pp. 1-43.

Marmon T., Kramer A. (2006), Ethnische Parteien der Andenländer:Von sozialen Bewegungen zu politischen Gestaltern, "German Institute of Global and Area Studies", no 11, pp. 2-9.

Martínez F. (2004), El primer partido político indígena en México, "Revista de Derecho y Cultura", no 13, pp. 103-116.

Misión de Observación Electoral de la Unión Europea en Paraguay (MOEUE 2013). Informe final (2013), Asunción.

North D. (1993), Instituciones, cambio institucional y desempeño económico, Fondo de Cultura Económica, México DF. 
Prieto E. (2013), Ciudadanía indígena en Paraguay, Universidad Técnica de Comercialización y Desarrollo (UTCD), Asunción.

Robins W. (1999), Etnicidad, tierra y poder, Centro de Estudios Antropológicos de la Universidad Católica (CEADUC), Asunción.

Sáenz de Tejada R. (2005), Elecciones, participación política y pueblo maya en Guatemala, Universidad Rafael Landivar, Guatemala.

Van Cott D. (2003), Cambio institucional y partidos étnicos en Sudamérica. "Revista Análisis Político", no 48, pp. 26-51.

Van Cott D. (2005), From Movements to Parties in Latin America. The Evolution of Ethnic Politics, Cambridge University Press, New York.

Villalba S. (2007), Organizaciones indígenas en Paraguay, "Suplemento Antropológico", vol. XLII, no 1, pp. 155-270.

Yashar D. (1998), Contesting citizenship. Indigenous movements and democracy in Latin America, "Comparative Politics", no 31, pp. 23-42.
Hacia nuevos retos.

Aproximación a la participación electoral indígena

\section{en Paraguay}

Sara Mabel Villalba Portillo 